\title{
Intraprocedural Cleansing for Screening Colonoscopy: Avoiding Brownouts
}

\author{
Felix W. Leung ${ }^{1,2}$
}

Received: 21 April 2015/Accepted: 23 April 2015/Published online: 2 June 2015

(C) Springer Science+Business Media New York (Outside the USA) 2015

Adequate bowel preparation has long been considered an essential prerequisite for successful endoscopic procedures since residual feces can obscure mucosal pathology [1], particularly flat polyps termed non-polypoid colorectal neoplasms (NP-CRNs) [2]. Overall, NP-CRNs are more likely to harbor carcinoma (odds ratio $\sim 10$ ) than polypoid lesions, irrespective of size [3]. With regard to shape, depressed-type NP-CRNs are associated with a $33 \%$ risk of malignancy, which is the highest of all polyp types [3]. Modifiable factors with an impact on adenoma detection rate (ADR) include increasing the length of time used for withdrawal inspection, looking behind folds, and retroflex examination for the detection of NP-CRNs. There is current emphasis regarding improving bowel cleanliness which improves recognition and facilitates complete resection of NP-CRNs, sessile serrated polyps, and advanced adenomas [4].

In this issue of the Digestive Diseases and Sciences, Oh et al. [5] evaluated the impact of the quality of bowel cleansing on detection of NP-CRNs. In a retrospective analysis of screening colonoscopy performed by seven board-certified attending gastroenterologists at one academic teaching hospital, patients underwent recommended split-dose bowel preparation, with endoscopic removal of fecal debris and retained fluid accomplished by forceful irrigation and suction, prior to rating bowel preparation

Felix W. Leung

felix.leung@va.gov

1 Gastroenterology, Sepulveda Ambulatory Care Center, Greater Los Angeles Veterans Affairs Healthcare System, 111G, 16111 Plummer Street, North Hills, CA 91343, USA

2 Gastroenterology Division, David Geffen School of Medicine, UCLA, Los Angeles, CA, USA quality. In 6097 asymptomatic, average-risk screening examinations, the preparation quality was rated as adequate (excellent or good) in 5224 (85.7\%), fair in 615 (10.1\%), and poor in $258(4.2 \%)$ patients. Efforts to improve the preparation quality increased the detection rate of NPCRNs, improving the efficacy of screening colonoscopies. Interestingly, the split-dose bowel preparation, formerly lauded for its efficacy on the basis of clinical trial data, was less perfect in real-life clinical settings, necessitating intraprocedural cleansing.

In the study by Oh et al. [5], the primary outcome was a comparison of the adenoma detection rate (ADR) for nonpolypoid lesions according to quality of bowel preparation. The overall ADR of non-polypoid pathology, $12.3 \%$ (747/ 6097) of all colonoscopies, significantly differed between participating endoscopists. The ADR for non-polypoid pathology was significantly lower if preparation was rated as fair or poor quality compared with the ADR associated with adequate preparation. Furthermore, detection of $<1 \mathrm{~cm}$ in lesions in the proximal colon was significantly lower with poor preparation than with adequate preparation. Of 5946 polyps detected in the study population, 4847 were histologically confirmed as adenomas $(84.7 \%)$ or serrated polyps $(15.3 \%)$. More than half were located in the proximal colon (2590/4847), most were of $<1 \mathrm{~cm}$ (4570/4847), and almost all were flat (0-IIa or 0-IIb) lesions (4843/4847). The proportion of NP-CRNs among all neoplastic lesions was $40.5 \%$ (1962/4847), with the proportion of advanced neoplasms among NP-CRNs $3.5 \%$ (68/1962).

Although the report is limited by its retrospective and single-site design, the study has several strengths: It involved a large patient population and also focused on NPCRNs which have recently assumed greater importance due to their increased malignant potential compared with 
conventional polypoid lesions. Strengths also included the participation of multiple colonoscopists, the inclusion of asymptomatic average-risk patients who underwent a firsttime screening colonoscopy, and the use of the recommended split-dose bowel preparation. Another unique feature was the application of clearing efforts to remove fecal debris and retained fluid by forceful irrigation and suction prior to rating of bowel preparation quality using the prevalent Aronchick scale. That suboptimal (fair or poor) bowel preparation was significantly associated with impaired detection of NP-CRNs combined with the finding of wide variability of ADRs among endoscopists confirmed prior similar reports. Development of competence among colonoscopists in the detection of NP-CRNs is hence a potential emphasis area.

This study reinforces current concerns regarding postscreening colonoscopy interval cancers by confirming that NP-CRNs can be easily missed as a result of inadequate colon preparation, supported by the observation that interval cancers cluster in the right colon (cecum and ascending colon) [6], traditionally recognized as the colonic segments most difficult to prepare [5]. Interval cancers are associated with colonoscopists with low ADRs [7]; the desirability of achieving high ADR is emphasized by the observation that a $1 \%$ increase in ADR reduces the overall risk of interval cancer by $3 \%$ and fatal cancers by $5 \%$ [8]. Notably, the conventional recommendation following colonoscopy with poor bowel preparation is a shorter screening or surveillance interval due to potential obscuration of smaller polyps.

Another contribution of the current report is to highlight the utility of intraprocedural bowel cleansing, a practical solution for patients with inadequate bowel preparation that could reduce the need for early repeat colonoscopy, thereby reducing costs, and patient and endoscopist time and inconvenience while potentially improving outcomes $[5,9$, 10]. Further data are needed to define the best means to clean the colon during an endoscopic procedure.

Endoscopists expending additional efforts to maximize cleansing of the bowel should never compromise the technique used or time spent to inspect the mucosa [11]. Conventional insertion techniques with air or carbon dioxide insufflation call for expeditious intubation of the cecum. Traditional intraprocedural cleansing is usually undertaken during withdrawal, which has not been questioned until recently as a distraction to the focused inspection for polyps. A novel method, insertional cleansing, is an integral component of the recently described method termed water-exchange colonoscopy $[12,13]$. Compared with traditional air insufflation in patients purged with split-dose bowel preparation, the novel technique has been associated with a significant increase in Boston Bowel Preparation scores and ADR in the right colon [12], and a significant reduction in distraction from focused examination during withdrawal [13]. Insertion cleaning, being relatively new, has not yet gained recognition as a component of standard insertion techniques. Nonetheless, its implementation is in line with the comment made by the authors of the current report that "...intraprocedural cleansing to enhance preparation quality could be a practical solution for patients with inadequate bowel preparation..." [5]. Whether wider adoption of insertion cleansing associated with water exchange will indeed lead to a reproducible increase in ADR and perhaps a reduction in the occurrence of interval cancers in the right colon remains to be studied in well-designed prospective clinical trials.

Acknowledgments Supported in part by VA Medical research funds.

Conflict of interest The author has no conflict of interest pertinent to this report to declare.

\section{References}

1. Chokshi RV, Hovis CE, Hollander T, et al. Prevalence of missed adenomas in patients with inadequate bowel preparation on screening colonoscopy. Gastrointest Endosc. 2012;75: 1197-1203.

2. Parra-Blanco A, Nicolas-Perez D, Gimeno-Garcia A, et al. The timing of bowel preparation before colonoscopy determines the quality of cleansing, and is a significant factor contributing to the detection of flat lesions: a randomized study. World $J$ Gastroenterol. 2006;12:6161-6166.

3. Soetikno RM, Kaltenbach T, Rouse RV, et al. Prevalence of nonpolypoid (flat and depressed) colorectal neoplasms in asymptomatic and symptomatic adults. JAMA. 2008;299: 1027-1035.

4. Tholey DM, Shelton CE, Francis G, et al. Adenoma detection in excellent versus good bowel preparation for colonoscopy. J Clin Gastroenterol. 2015;49:313-319.

5. Oh CH, Lee CK, Kim JW, et al. Suboptimal bowel preparation significantly impairs colonoscopic detection of nonpolypoid colorectal neoplasms. Dig Dis Sci. (Epub ahead of print). doi: 10. 1007/s10620-015-3628-6

6. Cooper GS, Xu F, Barnholtz Sloan JS, et al. Prevalence and predictors of interval colorectal cancers in medicare beneficiaries. Cancer. 2012;118:3044-3052.

7. Kaminski MF, Regula J, Kraszewska E, et al. Quality indicators for colonoscopy and the risk of interval cancer. $N$ Engl J Med. 2010;362:1795-1803.

8. Corley DA, Jensen CD, Marks AR, et al. Adenoma detection rate and risk of colorectal cancer and death. $N$ Engl J Med. 2014;370:1298-1306.

9. MacPhail ME, Hardacker KA, Tiwari A, et al. Intraprocedural cleansing work during colonoscopy and achievable rates of adequate preparation in an open-access endoscopy unit. Gastrointest Endosc. 2015;81:525-530.

10. Liang PS, Dominitz JA. Editorial: bowel preparation: is fair good enough? Am J Gastroenterol. 2014;109:1725-1727.

11. Calderwood AH, Thompson KD, Schroy PC 3rd, et al. Good is better than excellent: bowel preparation quality and adenoma detection rates. Gastrointest Endosc. 2015;81:691-699. 
12. Hsieh $\mathrm{YH}$, Koo M, Leung FW. A patient-blinded randomized, controlled trial (RCT) comparing air insufflation (AI), water immersion (WI) and water exchange (WE) during minimally sedated colonoscopy. Am J Gastroenterol. 2014;109:1390-1400.
13. Yen AW, Yung VY, Leung JW, et al. Insertion water exchange minimizes endoscopist multitasking during withdrawal inspection-a plausible explanation for enhanced polyp detection in the right colon. J Interv Gastroenterol. 2015;5:3-9. 